Supporting Information

\title{
Manipulating Hot-Electron Injection in Metal Oxide Heterojunction Array for Ultrasensitive Surface- Enhanced Raman Scattering
}

Xingce Fan*, Penghua Weit, Guoqun Li, Mingze Li, Leilei Lan, Qi Hao*, and Teng Qiu*

School of Physics, Southeast University, Nanjing 211189, China

*Email: tqiu@seu.edu.cn (T. Q.)

*Email: qihao@seu.edu.cn (Q. H.)

\$These authors contributed equally. 


\section{Supplementary Note I:}

\section{Fabrication and Characterization of Al Nanobowl Array Template}

High purity Al foils $(99.99 \%, 30 \times 30 \times 0.2 \mathrm{~mm})$ are ultrasonically cleaned in acetone and deionized water for $5 \mathrm{~min}$, respectively. Then, the $\mathrm{Al}$ foils are electrochemically polished in a mixture of ethanol and perchloric acid (volume ratio of 4:1) at a constant direct-current voltage of $15 \mathrm{~V}$ for $3 \mathrm{~min}$ to remove surface impurities.

For acquiring the ordered surface morphologies of templates, two-step anodization approach is adopted. The polished $\mathrm{Al}$ foils are anodized in $0.3 \mathrm{M}$ oxalic acid at $4{ }^{\circ} \mathrm{C}$ at constant direct-current voltage for $2 \mathrm{~h}$. The anodization voltage is denoted as $V_{A A O}$, which could be adjusted to tailor the unit size of Al nanobowl array template. The anodization voltage of 30, 40,50 and $60 \mathrm{~V}$ are adopted in this work. The anodic aluminum oxide (AAO) membrane grown during the first step is etched in a mixture of chromic acid and phosphoric acid (weight ratio of $1.8 \mathrm{wt} \%$ and $6 \mathrm{wt} \%$ ) at $75^{\circ} \mathrm{C}$ for $2 \mathrm{~h}$. Afterwards, the $\mathrm{Al}$ foils are subjected to the second anodization process with the same parameters as the first one. Finally, the Al nanobowl array templates are obtained after the removal of AAO membrane grown during the second step.

As shown in Figure S1a, the undulant surface morphology of Al nanobowl array template $\left(V_{\mathrm{AAO}}=\right.$ $40 \mathrm{~V})$ is demonstrated. The two-dimensional AFM image of Al nanobowl array template $\left(V_{\mathrm{AAO}}=40\right.$ $\mathrm{V}$ ) is present in Figure S1b, which shows a hexagonal unit arrangement. Each Al nanobowl is surrounded by six equivalent adjacent ones and can easily scale up to contiguous area of centimeter dimension. To describe the surface morphology more accurately, cross-section analysis along the blue and the violet lines labelled in Figure S1b are conducted. Periodic protrusions shown in Figures S1 (c, d) serve as "nano-containers" to assemble M/W-HA. 

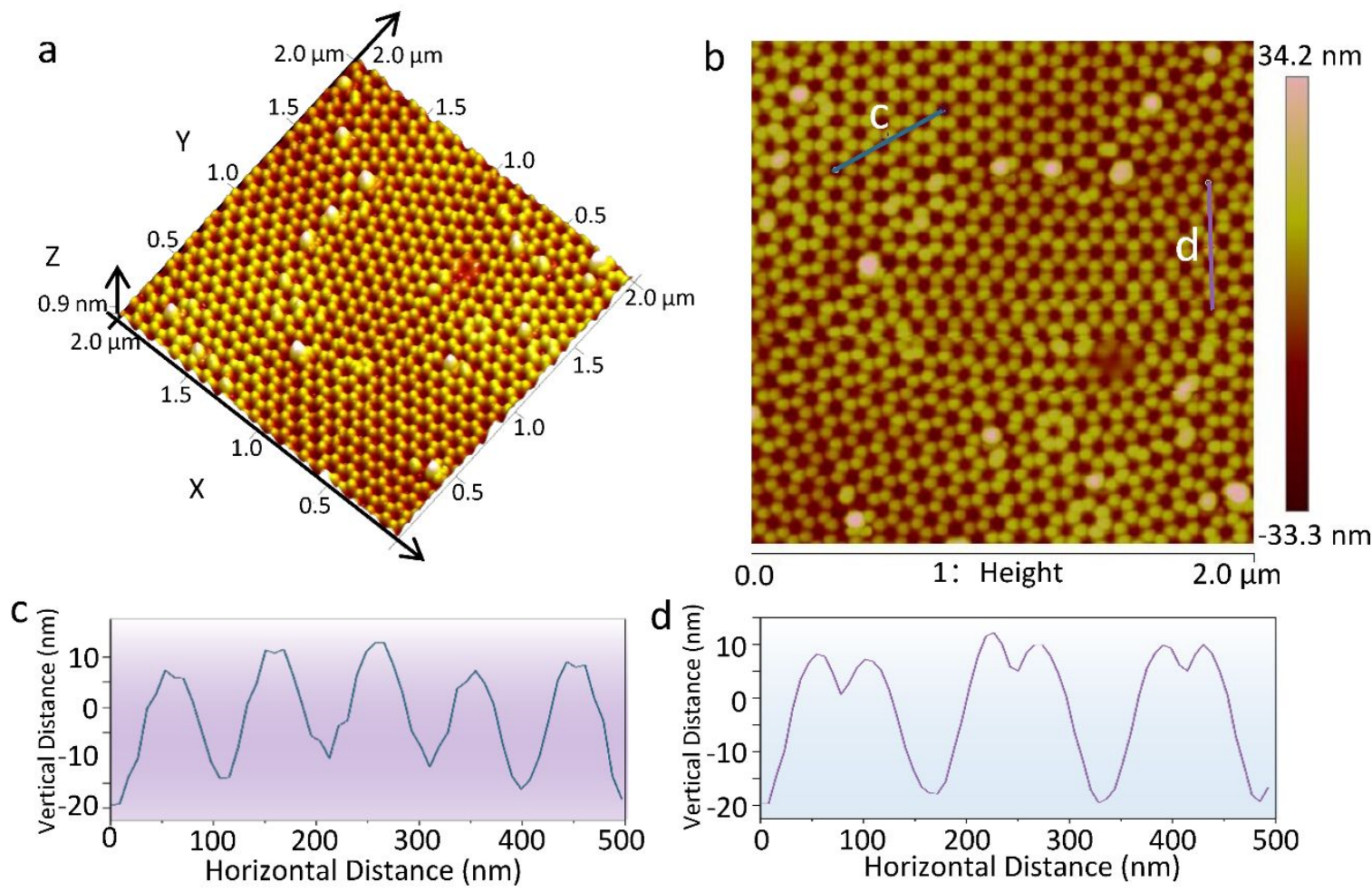

Figure S1. Characterizations of Al nanobowl array templates. (a) The three-dimensional and (b) two-dimensional AFM images of Al nanobowl array template $\left(V_{\mathrm{AAO}}=40 \mathrm{~V}\right) . \quad(\mathrm{c}, \mathrm{d})$ The cross-section views of Al nanobowl structure along the blue and violet lines marked in (b), respectively. 


\section{Supplementary Note II:}

\section{Characterizations of Planar-M/W and Planar-W (Reference Samples)}

As described in the main context, the reference samples planar-M/W and planar-W are fabricated on flat Al substrates, rather than on Al nanobowl array templates. The surface morphologies of reference samples are flat and smooth as shown in Figure S2. The corresponding element mappings (the inset of Figure S2) present uniform distributions of W, Mo, and O elements, confirming the composition homogeneity of reference samples.
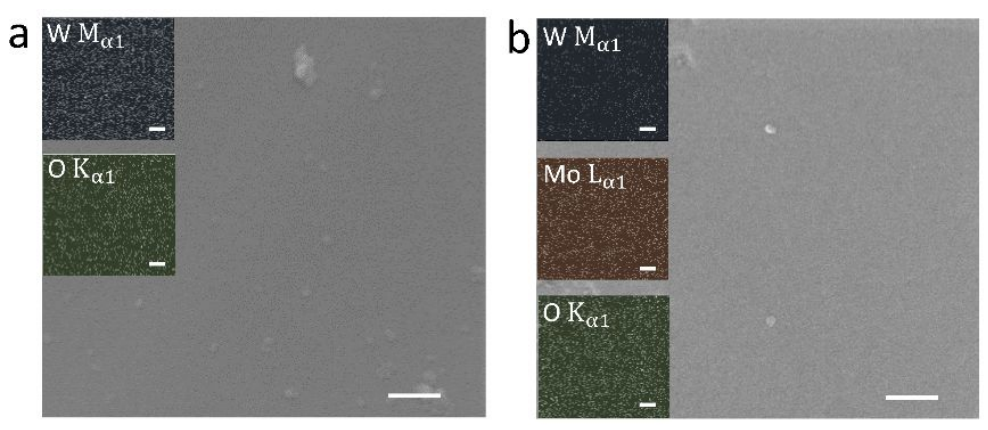

Figure S2. (a) SEM images and corresponding element mappings of (a) planar-W and (b) planar-M/W. Scalebars: $100 \mathrm{~nm}$.

a
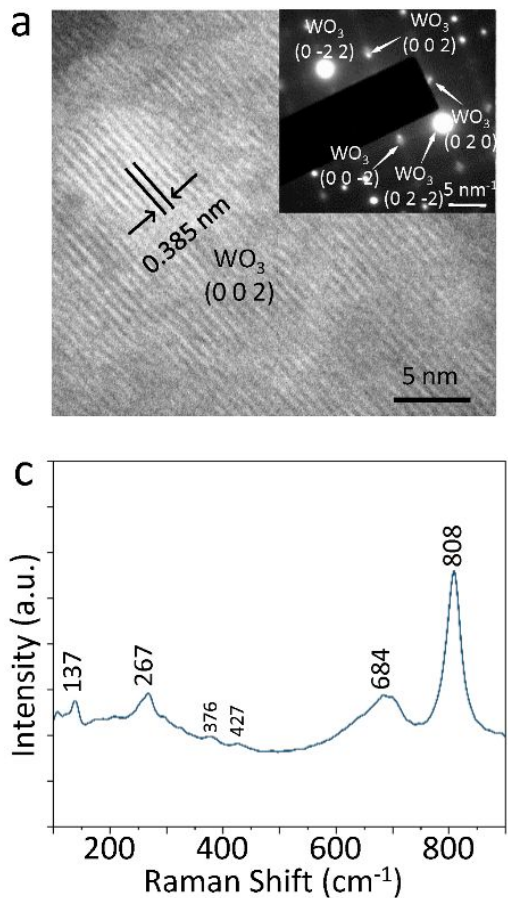
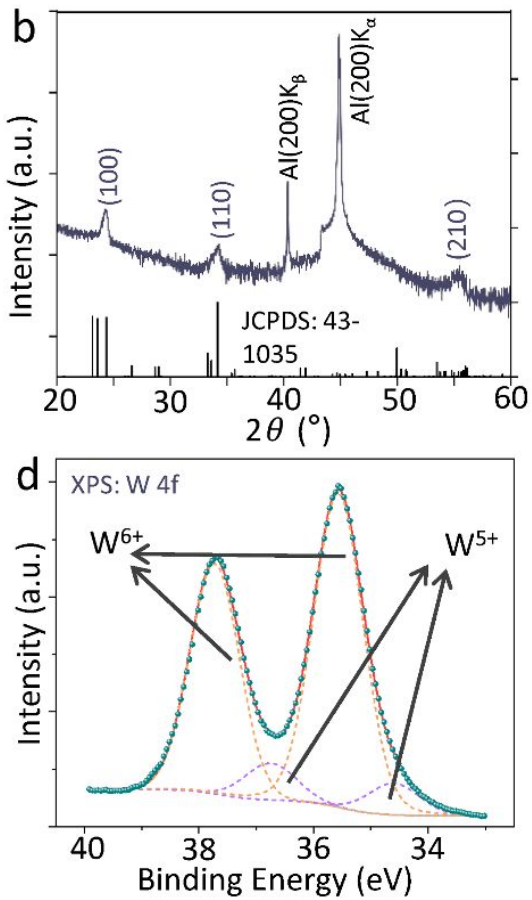

Figure S3. (a) HR-TEM image of planar-W. Inset: corresponding SAED pattern. (b) XRD, (c) Raman, and (d) XPS spectrum of planar-W. 


\begin{tabular}{|c|c|c|c|c|c|}
\hline Sample Atom & $\mathrm{W}^{6+}$ & $\mathrm{W}^{5+}$ & $\mathrm{Mo}^{6+}$ & $\mathrm{Mo}^{5+}$ & $\mathrm{Mo}^{4+}$ \\
\hline $\mathrm{M} / \mathrm{W}$ & $76.4 \%$ & $23.6 \%$ & $9.1 \%$ & $23.3 \%$ & $67.6 \%$ \\
\hline $\mathrm{W}$ & $77.8 \%$ & $22.2 \%$ & & & \\
\hline
\end{tabular}

Table S1. Atom percentage of $\mathrm{W}$ and $\mathrm{Mo}$ in $\mathrm{M} / \mathrm{W}-\mathrm{HA}$ and planar-W.

\begin{tabular}{|c|c|c|c|c|}
\hline $\begin{array}{l}\text { Excitation } \\
\text { Wavelength }(\mathrm{nm})\end{array}$ & \multirow{2}{*}{$\begin{array}{c}\mathrm{E}_{\max }^{6+} \\
(\mathrm{V} / \mathrm{m})\end{array}$} & $\mathrm{W}^{5+}$ & $\mathrm{Mo}^{6+}$ & $\mathrm{Mo}^{5+}$ \\
\cline { 1 - 4 } 532 & 8.17 & 7.26 & 5.71 & 5.05 \\
\hline 633 & 2.52 & 2.77 & 2.83 & 2.94 \\
\hline
\end{tabular}

Table S2. The maximum magnitudes of the local electric field in FDTD Simulations.

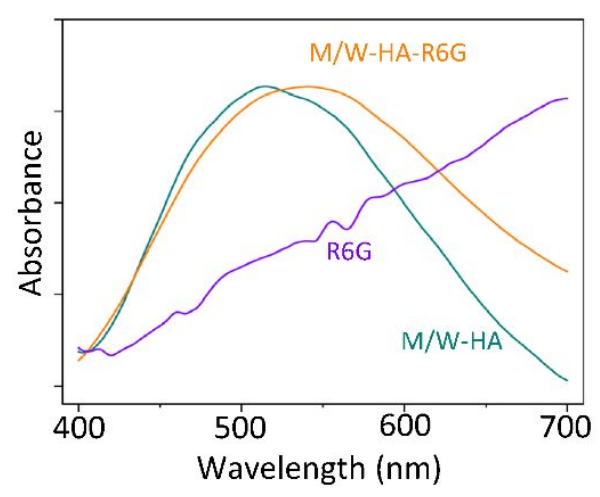

Figure S4. UV-vis spectra of M/W-HA-R6G, M/W-HA and R6G. 


\section{Supplementary Note III:}

\section{Calculation of Enhancement Factor}

The EF is calculated according to the following formula:

$$
\begin{aligned}
& \mathrm{EF}=\frac{I_{\text {SERS }} / N_{\text {SERS }}}{I_{\text {norm }} / N_{\text {norm }}} \\
& N_{\text {SERS }}=\frac{C V N_{A} A_{\text {Raman }}}{A_{\text {Sub }}} \\
& N_{\text {norm }}=\frac{\rho h A_{\text {Raman }} N_{A}}{M}
\end{aligned}
$$

$I_{S E R S}$ and $I_{\text {norm }}$ are the intensities of the selected Raman peak in SERS spectra and normal Raman spectra, respectively. $\quad N_{S E R S}$ and $N_{\text {norm }}$ are the number of molecules in the testing area of SERS and normal Raman measurements, respectively. The data of bulk R6G is used as normal Raman spectrum reference. For the SERS measurement, the intensity is obtained by taking average from measurements of 10 random spots, and the number of molecules is estimated by equation (S2) on the assumption that the molecules are distributed uniformly on the substrate. $C$ is the molar concentration of R6G solution and $V$ is the volume of the droplet $(10 \mu \mathrm{L}) . \quad N_{A}$ is Avogadro constant. $A_{\text {Raman }}$ is the laser spot area. $A_{\text {Sub }}$ is the effective area of the substrate, which is approximately $\pi / 4 \mathrm{~cm}^{2}$. The confocal depth $h$ of the laser beam is $\sim 21 \mu \mathrm{m}$. The molecular weight $M$ of R6G is $479 \mathrm{~g} \mathrm{~mol}^{-1}$ and density $\rho$ of bulk R6G is $1.15 \mathrm{~g} \mathrm{~cm}^{-3}$.

According to the above parameters, the calculated EF of M/W-HA is $\sim 2.12 \times 10^{8}$ at the R6G concentration of $1.0 \times 10^{-11} \mathrm{M}$. 


\section{Supplementary Note IV:}

Summary of Metal Oxide SERS Substrates

\begin{tabular}{|c|c|c|c|c|}
\hline SERS Substrates & Probe & $\operatorname{LoD}(\mathrm{M})$ & $\mathrm{EF}$ & Ref. \\
\hline $\mathrm{Li}_{\mathrm{x}} \mathrm{WO}_{3}$ film & R6G & $10^{-6}$ & $8.86 \times 10^{4}$ & 1 \\
\hline $\mathrm{W}_{18} \mathrm{O}_{49}$ nanoparticle & R6G & $10^{-7}$ & $3.4 \times 10^{5}$ & 2 \\
\hline $\mathrm{W}_{18} \mathrm{O}_{49}$ nanowire film & RhB & $10^{-7}$ & $4.4 \times 10^{5}$ & 3 \\
\hline $\mathrm{WO}_{3-\mathrm{x}}$ film & R6G & $10^{-7}$ & $1.1 \times 10^{4}$ & 4 \\
\hline $\mathrm{WO}_{3-\mathrm{x}}$ films & R6G & $10^{-9}$ & $1.16 \times 10^{6}$ & 5 \\
\hline $\mathrm{WO}_{2}$ nanodendrites & R6G & $10^{-10}$ & $8.5 \times 10^{7}$ & 6 \\
\hline $\mathrm{VO}_{2}$ nanosheets & R6G & $10^{-10}$ & $6.7 \times 10^{7}$ & 7 \\
\hline $\mathrm{W}_{18} \mathrm{O}_{49}$ nanowires & R6G & $10^{-11}$ & $1.2 \times 10^{7}$ & 8 \\
\hline $\mathrm{W}_{18} \mathrm{O}_{49}$ bundles & R6G & N/A & $2.8 \times 10^{7}$ & 9 \\
\hline Mo doped $\mathrm{Ta}_{2} \mathrm{O}_{5}$ nanowire & MV & $9 \times 10^{-9}$ & $2.2 \times 10^{7}$ & 10 \\
\hline Black $\mathrm{TiO}_{2}$ nanowire & R6G & $10^{-7}$ & $1.2 \times 10^{6}$ & 11 \\
\hline $\mathrm{TiO}_{2}$ nanoparticle & 4-MBA & $10^{-8}$ & N/A & 12 \\
\hline $\mathrm{TiO}_{2-\mathrm{x}} \mathrm{N}_{\mathrm{y}}$ bulk & R6G & $5 \times 10^{-8}$ & $4.5 \times 10^{5}$ & 4 \\
\hline$\gamma-\mathrm{Ti}_{3} \mathrm{O}_{5}$ microspheres & R6G & $10^{-10}$ & N/A & 13 \\
\hline Ni-doped $\mathrm{TiO}_{2}$ microarray & 4-MBA & $10^{-11}$ & $3.3 \times 10^{4}$ & 14 \\
\hline $\mathrm{CrO}_{\mathrm{x}}$ film & R6G & $10^{-9}$ & N/A & 15 \\
\hline $\mathrm{MoO}_{\mathrm{x}}$ film & R6G & $10^{-7}$ & $\mathrm{~N} / \mathrm{A}$ & 15 \\
\hline $\mathrm{MoO}_{3-\mathrm{x}}$ micron urchin & R6G & $10^{-7}$ & $\sim 10^{5}$ & 16 \\
\hline $\mathrm{MoO}_{3-\mathrm{x}}$ nanosheet & R6G & $10^{-7}$ & $3.32 \times 10^{5}$ & 17 \\
\hline $\mathrm{MoO}_{3-\mathrm{x}} @ \mathrm{MoO}_{3}$ nanosheets & MB & $10^{-7}$ & $1.42 \times 10^{5}$ & 18 \\
\hline $\mathrm{MoO}_{2}$ nanodumbbells & R6G & $10^{-7}$ & $3.75 \times 10^{6}$ & 19 \\
\hline $\mathrm{MoO}_{3-\mathrm{x}}$ nanobelts & R6G & $10^{-8}$ & $1.8 \times 10^{7}$ & 20 \\
\hline $\mathrm{MoO}_{2}$ nanosheets & R6G & $4 \times 10^{-8}$ & $2.1 \times 10^{5}$ & 21 \\
\hline $\mathrm{MoO}_{2}$ nanospheres & R6G & $10^{-8}$ & $4.8 \times 10^{6}$ & 22 \\
\hline
\end{tabular}




\begin{tabular}{|c|c|c|c|c|}
\hline $\mathrm{V}_{2} \mathrm{O}_{5}$ nanoparticle & R6G & $10^{-8}$ & $\mathrm{~N} / \mathrm{A}$ & 23 \\
\hline $\mathrm{VO}_{2}$ nanospheres & R6G & $10^{-9}$ & $\mathrm{~N} / \mathrm{A}$ & 24 \\
\hline $\mathrm{ZnO}$ nanocrystals & 4-Mpy & $10^{-5}$ & $10^{3}$ & 25 \\
\hline $\mathrm{ZnO}$ nanosheet & 4-Mpy & $10^{-7}$ & $7.7 \times 10^{5}$ & 26 \\
\hline ZnO QDs & R6G & $10^{-9}$ & $2.2 \times 10^{5}$ & 27 \\
\hline $\mathrm{Cu}_{2} \mathrm{O}$ nanospheres & 4-MBA & N/A & $10^{5}$ & 28 \\
\hline $\mathrm{Cu}_{2} \mathrm{O}$ concave sphere & R6G & $2 \times 10^{-8}$ & $2.8 \times 10^{5}$ & 29 \\
\hline $\mathrm{Cu}_{2} \mathrm{O}$ Superstructure particle & R6G & $10^{-9}$ & $8 \times 10^{5}$ & 30 \\
\hline amorphous $\mathrm{TiO}_{2}$ sheet & 4-MBA & $10^{-5}$ & $1.86 \times 10^{6}$ & 31 \\
\hline amorphous $\mathrm{ZnO}$ nanocage & 4-MBA & $10^{-5}$ & $6.62 \times 10^{5}$ & 32 \\
\hline $\mathrm{TiO} 2$ inverse opal & MB & $6 \times 10^{-6}$ & $2 \times 10^{4}$ & 33 \\
\hline $\mathrm{ZnO}$ superparticle & 4-Mpy & N/A & $10^{5}$ & 34 \\
\hline
\end{tabular}

\section{References}

1. Cong, S.; Wang, Z.; Gong, W.; Chen, Z.; Lu, W.; Lombardi, J. R.; Zhao, Z. Electrochromic Semiconductors as Colorimetric SERS Substrates with High Reproducibility and Renewability. Nat. Commun. 2019, 10, 678.

2. Cong, S.; Yuan, Y.; Chen, Z.; Hou, J.; Yang, M.; Su, Y.; Zhang, Y.; Li, L.; Li, Q.; Geng, F.; Zhao, Z. Noble Metal-Comparable SERS Enhancement from Semiconducting Metal Oxides by Making Oxygen Vacancies. Nat. Commun. 2015, 6, 7800.

3. Wang, X.; Li, J.; Shen, Y.; Xie, A. An Assembled Ordered $\mathrm{W}_{18} \mathrm{O}_{49}$ Nanowire Film with High SERS Sensitivity and Stability for the Detection of RB. Appl. Surf. Sci. 2020, 504, 144073.

4. Zheng, X.; Ren, F.; Zhang, S.; Zhang, X.; Wu, H.; Zhang, X.; Xing, Z.; Qin, W.; Liu, Y.; Jiang, C. A General Method for Large-scale Fabrication of Semiconducting Oxides with High SERS Sensitivity. ACS Appl. Mater. Interfaces 2017, 9, 14534 - 14544.

5. Fan, X.; Li, M.; Hao, Q.; Zhu, M.; Hou, X.; Huang, H.; Ma, L.; Schmidt, O. G.; Qiu, T. High SERS Sensitivity Enabled by Synergistically Enhanced Photoinduced Charge Transfer in Amorphous 
Nonstoichiometric Semiconducting Films. Adv. Mater. Interfaces 2019, 6, 1901133.

6. Ye, Y.; Chen, C.; Bai, H.; Liu, W.; Li, W.; Li, J.; Yi, W.; Xi, G. Quasi-metallic Tungsten Oxide Nanodendrites with High Stability for Surface-enhanced Raman Scattering. Cell Rep. Phys. Sci. 2020, $1,100031$.

7. Tian, Z.; Bai, H.; Chen, C.; Ye, Y.; Kong, Q.; Li, Y.; Fan, W.; Yi, W.; Xi G. Quasi-Metal for Highly Sensitive and Stable Surface-enhanced Raman Scattering. iScience 2019, 19, 836 - 849.

8. Ye, Y.; Chen, C.; Li, W.; Guo, X.; Yang, H.; Guan, H.; Bai, H.; Liu, W.; Xi, G. Highly Sensitive $\mathrm{W}_{18} \mathrm{O}_{49}$ Mesocrystal Raman Scattering Substrate with Large-Area Signal Uniformity. Anal. Chem. 2021, 93, $3138-3145$.

9. Liu, W.; Bai, H.; Li, X.; Li, W.; Zhai, J.; Li, J.; Xi, G. Improved Surface-enhanced Raman Spectroscopy Sensitivity on Metallic Tungsten Oxide by the Synergistic Effect of Surface Plasmon Resonance Coupling and Charge Transfer. J. Phys. Chem. Lett. 2018, 9, 4096 - 4100.

10. Yang, L.; Peng, Y.; Yang, Y.; Liu, J.; Huang, H.; Yu, B.; Zhao, J.; Lu, Y.; Huang, Z.; Li, Z.; Lombardi, J. R. A Novel Ultra-Sensitive Semiconductor SERS Substrate Boosted by the Coupled Resonance Effect. Adv. Sci. 2019, 6, 1900310.

11. Yang, L.; Peng, Y.; Yang, Y.; Liu, J.; Li, Z.; Ma, Y.; Zhang, Z.; Wei, Y.; Li, S.; Huang, Z.; Long, N. V. Green and Sensitive Flexible Semiconductor SERS Substrates: Hydrogenated Black $\mathrm{TiO}_{2}$ Nanowires. ACS Appl. Nano Mater. 2018, 1, 4516 - 4527.

12. Yang, L.; Yin, D.; Shen, Y.; Yang, M.; Li, X.; Han, X.; Jiang, X.; Zhao, B. Mesoporous Semiconducting $\mathrm{TiO}_{2}$ with Rich Active Sites as a Remarkable Substrate for Surface-Enhanced Raman Scattering. Phys. Chem. Chem. Phys. 2017, 19, $18731-18738$.

13. Li, Y.; Bai, H.; Zhai, J.; Yi, W.; Li, J.; Yang, H.; Xi, G. Alternative to Noble Metal Substrates: Metallic and Plasmonic $\mathrm{Ti}_{3} \mathrm{O}_{5}$ Hierarchical Microspheres for Surface Enhanced Raman Spectroscopy. Anal. Chem. 2019, 91, 4496-4503.

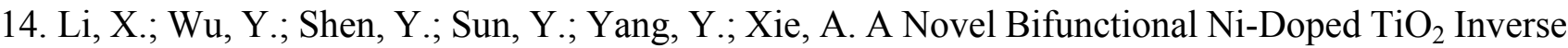
Opal with Enhanced SERS Performance and Excellent Photocatalytic Activity. Appl. Surf. Sci. 2018, 427, $739-744$.

15. Hou, X.; Fan, X.; Wei, P.; Qiu, T. Planar Transition Metal Oxides SERS Chips: A General Strategy. 
J. Mater. Chem. C 2019, 7, $11134-11141$.

16. Prabhu, B. R.; Bramhaiah, K.; Singh, K. K.; John, N. S. Single Sea Urchin-MoO $\mathrm{M}_{3}$ Nanostructure for Surface Enhanced Raman Spectroscopy of Dyes. Nanoscale Adv. 2019, 1, 2426 - 2434.

17. Lan, L.; Hou, X.; Gao, Y.; Fan, X.; Qiu, T. Inkjet-Printed Paper-Based Semiconducting Substrates for Surface-Enhanced Raman Spectroscopy. Nanotechnology 2020, 31, 055502.

18. Tan, X.; Wang, L.; Cheng, C.; Yan, X.; Shen, B.; Zhang, J. Plasmonic $\mathrm{MoO}_{3-\mathrm{x}} @ \mathrm{MoO}_{3} \mathrm{Nanosheets}$ for Highly Sensitive SERS Detection through Nanoshell-Isolated Electromagnetic Enhancement. Chem. Commun. 2016, 52, 2893 - 2896.

19. Zhang, Q.; Li, X.; Ma, Q.; Zhan,g Q.; Bai, H.; Yi, W.; Liu, J.; Han, J.; Xi, G. A Metallic Molybdenum Dioxide with High Stability for Surface Enhanced Raman Spectroscopy. Nat. Commun. 2017, 8, 14903.

20. Wu, H.; Wang, H.; Li, G. Metal Oxide Semiconductor SERS-Active Substrates by Defect Engineering. Analyst 2017, 142, $326-335$.

21. Wu, H.; Zhou, X.; Li, J.; Li, X.; Li, B.; Fei, W.; Zhou, J.; Yin, J.; Guo, W. Ultrathin Molybdenum Dioxide Nanosheets as Uniform and Reusable Surface-Enhanced Raman Spectroscopy Substrates with High Sensitivity. Small 2018, 14, 1802276.

22. Zhang, Q.; Li, X.; Yi, W.; Li, W.; Bai, H.; Liu, J.; Xi, G. Plasmonic $\mathrm{MoO}_{2}$ Nanospheres as a Highly Sensitive and Stable Non-Noble Metal Substrate for Multicomponent Surface-Enhanced Raman Analysis. Anal. Chem. 2017, 89, 11765 - 11771.

23. Pan, J.; Li, M.; Luo, Y.; Wu, H.; Zhong, L.; Wang, Q.; Li, G. Synthesis and SERS Activity of $\mathrm{V}_{2} \mathrm{O}_{5}$ Nanoparticles. Appl. Surf. Sci. 2015, 333, $34-38$.

24. Guan, H.; Tian, Z.; Kong, Q.; Xi, G. Vanadium Dioxide Nanostructures with Remarkable SurfaceEnhanced Raman Scattering Activity. Chem. Commun. 2021, 57, 4815 - 4818.

25. Wang, Y.; Ruan, W.; Zhang, J.; Yang, B.; Xu, W.; Zhao, B.; Lombardi, J. R. Direct Observation of Surface-Enhanced Raman Scattering in ZnO Nanocrystals. J. Raman Spectrosc. 2009, 40, 1072 $-1077$.

26. Lin, J.; Yu, J.; Akakuru, O. U.; Wang, X.; Yuan, B.; Chen, T.; Guo L.; Wu, A. Low TemperatureBoosted High Efficiency Photo-Induced Charge Transfer for Remarkable SERS Activity of ZnO 
Nanosheets. Chem. Sci. 2020, 11, $9414-9420$.

27. Haldavnekar, R.; Venkatakrishnan, K.; Tan, B. Non-Plasmonic Semiconductor Quantum SERS Probe as a Pathway for In Vitro Cancer Detection. Nat. Commun. 2018, 9, 3065.

28. Jiang, L.; You, T.; Yin, P.; Shang, Y.; Zhang, D.; Guo, L.; Yang, S. Surface-Enhanced Raman Scattering Spectra of Adsorbates on $\mathrm{Cu}_{2} \mathrm{O}$ Nanospheres: Charge-Transfer and Electromagnetic Enhancement. Nanoscale 2013, 5, $2784-2789$.

29. Li, X.; Shang, Y.; Lin, J.; Li, A.; Wang, X.; Li, B.; Guo, L. Temperature-Induced Stacking to Create $\mathrm{Cu}_{2} \mathrm{O}$ Concave Sphere for Light Trapping Capable of Ultrasensitive Single-Particle Surface-Enhanced Raman Scattering, Adv. Funct. Mater. 2018, 28, 1801868.

30. Lin, J.; Shang, Y.; Li, X.; Yu, J.; Wang, X.; Guo, L. Ultrasensitive SERS Detection by Defect Engineering on Single $\mathrm{Cu}_{2} \mathrm{O}$ Superstructure Particle, Adv. Mater. 2017, 29, 1604797.

31. Wang, X.; Shi, W.; Wang, S.; Zhao, H.; Lin, J.; Yang, Z.; Chen, M.; Guo, L. Two-Dimensional Amorphous $\mathrm{TiO}_{2}$ Nanosheets Enabling High-Efficiency Photoinduced Charge Transfer for Excellent SERS Activity, J. Am. Chem. Soc. 2019, 141, 5856 - 5862.

32. Wang, X.; Shi, W.; Jin, Z.; Huang, W.; Lin, J.; Ma, G.; Li, S.; Guo, L. Remarkable SERS Activity Observed from Amorphous ZnO Nanocages, Angew. Chem. Int. Ed. 2017, 129, 9983 - 9987.

33. Qi, D.; Lu, L.; Wang, L.; Zhang, J. Improved SERS Sensitivity on Plasmon-Free $\mathrm{TiO}_{2} \mathrm{Photonic}$ Microarray by Enhancing Light-Matter Coupling, J. Am. Chem. Soc. 2014, 136, 9886 - 9889.

34. Ji, W.; Li, L.; Song, W.; Wang, X.; Zhao, B.; Ozaki, Y. Enhanced Raman Scattering by ZnO Superstructures: Synergistic Effect of Charge Transfer and Mie Resonance, Angew. Chem. Int. Ed. 2019, $58,14452-14456$. 